\title{
An Analysis of Dynamic Vibrations: The Invariant Normal Curvature Wave Equation
}

\author{
Keith C. Afas ${ }^{* 1}$ and Terry E. Moschandreou ${ }^{\dagger 2}$ \\ ${ }^{2}$ Department of Applied Mathematics, Western University, London, \\ Ontario, N6A 5B7,Canada
}

September 25, 2017

\section{Abstract}

This paper attempts to address the phenomenon of normal vibrations, (referred to as dynamic vibrations) occurring on a surface which is already in vibrational motion due to other kinematic phenomena. Such a surface will have a metric tensor, normal, and ambient velocity which diverges from the surface's original various dynamic tensorial descriptors. This paper formulates the wave equation defined in a coordinate space, and extends the equation to observe vibrations on a surface with the use of the Laplace-Beltrami operator in a tensorial fashion drawing on conventions from the newly established Calculus of Moving Surfaces (CMS). The Paper then identifies the way which these normal vibrations will manifest within ambient space. Finally, a counter-intuitive relation between the magnitude of such dynamic vibrations and dynamic surface's time-dependent mean curvature presents itself, for which dynamic

\footnotetext{
*E-mail address: kafas@uwo.ca

${ }^{\dagger}$ Corresponding author, E-mail address: tmoschan@uwo.ca
} 
vibrations superimposed on original dynamic motion will eliminate the other, and the surface remains static under an arbitrary initial motion. From this condition, resubstitution within the wave equation yields a novel coupled PDE system which within contains the time-evolution of the surface's mean curvature and its dynamic vibrations. This analysis can have application for algorithms designed for mechanical stabilization during seismic activity, as well as analyzing stabilization algorithms for other various applications and can also have application with respect to biotechnological innovations for analyzing properties defined on the surface of cell-Like biological entities such as metabolism, lipid content, and actin dynamics.

\section{Introduction}

\subsection{The Problem at Hand}

Under seismic activity, earth is known to transmit transverse waves along the surface. These seismic Waves though large in magnitude have very little spatial variation. This is because of the sheer order of magnitude of the size of earth. However, in order to counter-balance the vibrations thus creating innovational algorithms which render mechanical constructions stable under seismic activity, it is demonstrated that an understanding of the method by which vibrations propagate on already dynamic surface is essential.

On another nano-order of magnitude, especially with the fast-growing field of nano-technology, it is often desired to be able to design calibrated instruments which can successfully attach and obtain measurements which pertain to the surface of the cell such as metabolism, ligand-receptor binding affiinities, and viral RNA transcript fluxes. These instruments would have unsuccessful measurements if the underlying surface is under extreme motion as cells often are. Thus the innovation of an algorithm by which a nano-functional machine can use to stabilize the cell [6] to an definite order 
of magnitude is required in order to obtain successful measurements.

\subsection{Right Tool for the Job}

In both of the above examples (more so for the cellular example) the issue at hand deals with stabilizing vibrations which are occurring on a surface that is already dynamic $[2-5,7,8,9,10]$ (thus aptly referred to as dynamic vibrations) which in the cellular case are due to actin dynamics, bulk motion, and shear forces. Since in either case, there is no definite preferred geometry, the analysis of dynamic vibrations requires a foundation which is fundamentally tensorial and also explicitly designed to analyze surfaces in a compact manner

The analysis of dynamic vibrations is naturally a CMS subject; metric tensors and surface velocities are going to be position dependent, and therefore naturally, CMS objects defined on moving surfaces. More importantly, formulating the problem in a tensorial frame is useful. By formulating according to CMS conventions, certain geometric properties can arise in a more compact form than normal and are also valid

in various coordinate systems which ultimately reveals fundamental properties of the surface understandable from an intuitive viewpoint.

Before an Analysis of the surface can be made, a constraint is already imposed on the problem. Any dynamic vibrations defined on a dynamic surface or a static one are going to be directed in the direction of the surface's normal and must obey the wave equation which might be operating on a surface that possesses curvature. Typically the wave equation is defined to operate on coordinate maps and on ambient spaces; this means that the wave equation must be formulated to accommodate this curvilinear change in environment. This change in environment is solved by using the Laplace-Beltrami operator 


\subsection{Wave Equation}

\section{Standard Wave Equation}

We consider the standard wave equation defining waves on a certain domain in a coordinate map. Any displacement of the coordinate map is denoted by $\psi\left(Z^{1}, Z^{2}, \ldots, Z^{i}, t\right)$, where $Z^{i}$ are the coordinates on the coordinate map that $\psi$ is defined on.

This equation is usually formulated in the form of:

$$
\square \psi=0
$$

where the $\square$ operator, also known as the D'Alembert Operator, is in the tensor calculus form of:

$$
\square=\frac{1}{c^{2}} \frac{\partial^{2}}{\partial t^{2}}-\nabla_{i} \nabla^{i}
$$

In this definition, the indices adhere to the Einstein summation convention. Here, we define $\nabla_{i}$ to be the covariant derivative in the coordinate system chosen to define $\psi$ on.

We also by convention allow $\nabla^{i}=Z^{i j} \nabla_{j}$ to be the contravariant derivative in the respective coordinate system, where $Z_{i j}$ is the metric tensor of the coordinate system, and $Z^{i j}$ is its inverse. Thus, the $\nabla_{i} \nabla^{i}$ term is known as the Laplacian operator. Notice how the form of the Laplacian depends entirely on the coordinate system chosen.

In these cases the Laplacian term can almost always be given explicitly by using the Voss-Weyl formula[1]:

$$
\nabla_{k} \nabla^{k}=\frac{1}{\sqrt{\left|Z_{i j}\right|}} \frac{\partial}{\partial Z^{k}}\left(\sqrt{\left|Z_{i j}\right|} \nabla^{k}\right)=\frac{1}{\sqrt{\left|Z_{i j}\right|}} \frac{\partial}{\partial Z^{k}}\left(\sqrt{\left|Z_{i j}\right|} Z^{k \ell} \nabla_{\ell}\right)
$$


The important part to notice about this formulation of the wave equation is that it is entirely dependent on the form of the Laplacian, which is in turn dependent on the surface of the coordinate system that $\psi$ is defined on. This method loses some of the wanted generality when analyzing waves on surfaces. For instance:

- waves on a section in ambient space with Cartesian geometry have an identity metric tensor, and thus will have the D'Alembert Operator of the form:

$$
\square=\frac{1}{c^{2}} \frac{\partial^{2}}{\partial t^{2}}-\sum_{i=1}^{2} \frac{\partial^{2}}{\partial\left(Z^{i}\right)^{2}}
$$

where $Z^{i}=\left(Z^{1}, Z^{2}, \ldots, Z^{i}\right)=(x, y)$ are the coordinates of the region. This coincides with our knowledge of the wave equation

- waves on a section in Ambient Space with polar geometry $(r, \theta)$ will have the following metric:

$$
Z_{i j}=\left[\begin{array}{ll}
1 & 0 \\
0 & r^{2}
\end{array}\right]
$$

which will produce an D'Alembert operator of:

$$
\square=\frac{1}{c^{2}} \frac{\partial^{2}}{\partial t^{2}}-\frac{1}{r} \frac{\partial}{\partial r}\left(r \frac{\partial}{\partial r}\right)-\frac{1}{r^{2}} \frac{\partial^{2}}{\partial \theta^{2}}
$$

This too, coincides with our knowledge of the wave equation in polar coordinates

Though this is effective for creating wave equation on coordinate maps, we need an equivalent method for describing vibrations on a surface which is also dynamic [2]

\section{Another way of looking at the wave equation}

In the previous section, we used $Z^{i}$ to refer to the ambient coordinates of the coordinate map which we defined $\psi$ on. Here we consider a surface with a parametrization:

$$
Z^{i}=Z^{i}\left(S^{1}, S^{2}, \ldots, S^{\alpha}, t\right)
$$


Here we define $Z^{i}$ as the ambient coordinates of the region, and we denote $S^{\alpha}$ as the surface coodinates. In some cases, the surface is referred to from an arbitrary origin point by the following 'position' vector, $\mathbf{R}=Z^{i} \mathbf{Z}_{\mathbf{i}}$. In general, all indices in tensors pertaining to surface coordinates are given greek characters $(\alpha, \beta, \gamma \ldots)$. When relating the surface to the ambient coordinates, we derive the Surface tangent vectors defined as:

$$
\mathbf{S}_{\alpha}=\frac{\partial \mathbf{R}}{\partial S^{\alpha}}
$$

The tangent vectors are referrable to the ambient space through the Shift Tensor, $Z^{i}{ }_{\alpha}$ where $\mathbf{S}_{\alpha}=Z^{i}{ }_{\alpha} \mathbf{Z}_{i}$. Such a surface has a metric given by:

$$
S_{\alpha \beta}=Z_{i j} Z_{\alpha}^{i} Z_{\beta}^{j}
$$

and a unit normal field defined by:

$$
\mathbf{N}=\frac{1}{2} \mathbf{Z}^{i} \epsilon_{i j k} \epsilon^{\alpha \beta} Z_{\alpha}^{j} Z_{\beta}^{k}
$$

Where $\epsilon_{i j k}$ is the ambient Levi-Civita Symbol, and $\epsilon_{\alpha \beta}$ is the surface Levi-Civita Symbol. We notice that such a metric automatically induces a covariant derivative, $\nabla_{\alpha}$. Establishing a covariant derivative, the surface Laplacian naturally follows giving:

$$
\nabla_{\gamma} \nabla^{\gamma}=\frac{1}{\sqrt{\left|S_{\alpha \beta}\right|}} \frac{\partial}{\partial S^{\gamma}}\left(\sqrt{\left|S_{\alpha \beta}\right|} \nabla^{\gamma}\right)=\frac{1}{\sqrt{\left|S_{\alpha \beta}\right|}} \frac{\partial}{\partial S^{\gamma}}\left(\sqrt{\left|S_{\alpha \beta}\right|} S^{\gamma \delta} \nabla_{\delta}\right)
$$

The benefit of such a definition is the we an eliminate coodinate maps entirely, and focus on vibrations defined on, very real, surfaces. With a surface Laplacian defined, we can define the surface D'Alembert operator as:

$$
\tilde{\square}=\frac{1}{c^{2}} \frac{\partial^{2}}{\partial t^{2}}-\nabla_{\alpha} \nabla^{\alpha}
$$


Here, displacements are given by the symbol $\overrightarrow{\boldsymbol{\sigma}}$ which can be decomposed with respect to the orthogonal basis frame $\sigma^{\mu} \boldsymbol{\xi}_{\mu}=\sigma^{\alpha} S_{\alpha}+\sigma^{3} \mathbf{N}$. We now state a reformulation of the equation as the surface wave equation:

$$
\tilde{\square}|\overrightarrow{\boldsymbol{\sigma}}|=0
$$

In this, $\vec{\sigma}$ indicates the magnitude of any displacements of the surface points, indexed by the surface coordinates. This definition, also coincides with the definition given previously:

- If we consider a standard square surface, $\Sigma$, we notice that its parametrization is given by:

$$
\Sigma: \begin{cases}x=x & \\ y=y, & x \in[a . . b] \\ z=0 & y \in[c . . d]\end{cases}
$$

Here, we note that $Z^{i}=\left(Z^{1}, Z^{2}, Z^{3}\right)=(x, y, z)$, and $S^{\alpha}=\left(S^{1}, S^{2}\right)=(x, y)$.

This surface has a Shift Tensor, Metric, and Normal given by

$$
Z_{\alpha}^{i}=\left[\begin{array}{ll}
1 & 0 \\
0 & 1 \\
0 & 0
\end{array}\right] \quad S_{\alpha \beta}=\left[\begin{array}{ll}
1 & 0 \\
0 & 1
\end{array}\right] \quad N_{i}=\left[\begin{array}{l}
0 \\
0 \\
1
\end{array}\right]
$$

Interestingly, the surface metric, much like the ambient metric tensor in the last section are identical. This results in an identical covariant derivative, and identical PDE. Worth noting, any displacements on the surface are going to be in the direction of the normal (here, in the z-direction) this is intuitive as $|\overrightarrow{\boldsymbol{\sigma}}|$, is commonly denoted as $z=|\overrightarrow{\boldsymbol{\sigma}}|(x, y)$.

- If we consider a standard circle surface, $\Sigma$, we notice that its parametrization is 
given by:

$$
\Sigma:\left\{\begin{array}{cc}
x=r \cos \theta & \\
y=r \sin \theta, & r \in[0 . . a] \\
z=0 & \theta \in[0 . .2 \pi]
\end{array}\right.
$$

Here, we note that $Z^{i}=\left(Z^{1}, Z^{2}, Z^{3}\right)=(x, y, z)$, and $S^{\alpha}=\left(S^{1}, S^{2}\right)=(r, \theta)$.

This surface has a shift tensor, metric, and normal given by

$$
Z_{\alpha}^{i}=\left[\begin{array}{cc}
\cos \theta & -r \sin \theta \\
\sin \theta & r \cos \theta \\
0 & 0
\end{array}\right] \quad S_{\alpha \beta}=\left[\begin{array}{cc}
1 & 0 \\
0 & r^{2}
\end{array}\right] \quad N_{i}=\left[\begin{array}{l}
0 \\
0 \\
1
\end{array}\right]
$$

Interestingly, the surface metric, much like the ambient metric tensor in the last section for a circle are identical. This results in an identical covariant derivative, and identical PDE. Worth noting, any displacements on the surface are going to be in the direction of the normal (here, in the z-direction) this is intuitive as $|\overrightarrow{\boldsymbol{\sigma}}|$, is commonly denoted as $z=|\overrightarrow{\boldsymbol{\sigma}}|(r, \theta)$

Thus we see that the equations are in fact, identical formulations. The biggest difference is surface metric tensors may be computed with ease for arbitrary surfaces and thus wave equations can be defined on any surface including dynamic surfaces. In general for curvilinear $3 \mathrm{~d}$ surfaces, the vibration mechanism may not always be in the normal and so we must consider the full general $\overrightarrow{\boldsymbol{\sigma}}$.

\subsection{Motivation}

Now that vibrations on surfaces are established to be given by:

$$
\tilde{\square}|\overrightarrow{\boldsymbol{\sigma}}|=0
$$


The embedding of the waves within the ambient space can now be analyzed.

\section{Embedding Waves in Ambient Space}

Another important part of solving the equation is how to embed the solution of the equation in ambient space. When dealing with coordinate maps, the issue is resolved as the Normal will always be in the z-direction, and thus be representable in the form $z=\psi$

Coordinate Maps are special cases of a surface where the Normal is in the zdirection. When dealing with 3-D surfaces, this assumption of the Normal's direction vanishes. Also additional considerations arise:

- With coordinate maps, it was always noted how $z=0$. This resulted in the new parametrization of the surface to be of the form $z=|\overrightarrow{\boldsymbol{\sigma}}|(S)$. In many scenarios, this is not the case (for example, vibrations on a sphere).

- With the vibrations defined, a dynamic parametrization may be obtained describing the way that the dynamic surface containing dynamic vibrations will move.[2] We denote this new parametrization as $\mathbf{R}^{*}$.

- In some cases, a surface with vibrations defined on it, will also moving dynamically, pre-vibration. In this case both the current and new post-vibration parametrization will have to be time dependent.

With all these considerations, we attempt to generalize the post-vibration parametrization.

\section{Creating the Static Frame}

Any deformed surface possess two surface unit tensors, $\boldsymbol{S}_{\alpha}$. For these two surface tensors a third tensor is defined which is orthogonal to the other two surface tensors. This 
tensor is commonly known as the normal and is explicitly given by:

$$
N=S_{1} \times S_{2}
$$

This may be formulated into a component by component form:

$$
N_{i}=\frac{1}{2} \epsilon_{i j k} \epsilon^{\alpha \beta} Z_{\alpha}^{j} Z_{\beta}^{k}
$$

such that $\boldsymbol{N}=N_{i} \boldsymbol{Z}^{i}, \epsilon_{i j k}$ is the ambient Levi-Civiita Symbol and $\epsilon_{\alpha \beta}$ is the surface Levi-Civita Symbol.

$\boldsymbol{S}_{\alpha}$ and $\boldsymbol{N}$ define the Static Frame which has an intrinsically geometric significance and is exactly the local Euclidean space which is rotated to be tangent with the surface. Every surface will have its own intrinsic static frame.This frame is assumed to be composed of a curvilinear varying basis, denoted by $\boldsymbol{\xi}_{\mu}$. Using this basis, we can define any tensor from a surface's apex. Suppose we let $\boldsymbol{T}$ be a tensor. If we express this in terms of the local coordinate basis, we see that:

$$
\boldsymbol{T}=T^{\mu} \boldsymbol{\xi}_{\mu}=T^{\alpha} \boldsymbol{S}_{\alpha}+T^{3} \boldsymbol{N}
$$

An interesting aspect of this decomposition is the reduction for a covariant tensor:

$$
\boldsymbol{T}=T_{\mu} \boldsymbol{\xi}^{\mu}=T^{\alpha} \boldsymbol{S}_{\alpha}+T_{3} \boldsymbol{N}
$$

\section{Vibrating Surface Parametrization}

With these, a new parametrization is proposed as the following:

$$
\mathbf{R}^{*}=\mathbf{R}+\sigma^{\mu} \boldsymbol{\xi}_{\mu}
$$


Accounting for the dynamic nature of the problem, we also allow everything to have a dependency on time:

$$
\mathbf{R}^{*}(S, t)=\mathbf{R}(S, t)+\sigma^{\mu}(S, t) \boldsymbol{\xi}_{\mu}(S, t)
$$

Computing the surface speed, normal, and metric of such a surface[1] is important for predicting changes to the surface, understanding the nature of Dynamic Vibrations, and one focus of the paper.

\section{Covariant Derivatives in the Static Frame}

From Eq.(16) we define the following in-surface base vectors as [11]:

$$
\begin{gathered}
\boldsymbol{a}_{\alpha}=\boldsymbol{R},_{\alpha} \\
\boldsymbol{g}_{\alpha}=\left(\boldsymbol{R}^{*},{ }_{\alpha}-\boldsymbol{a}_{\alpha}\right)=\boldsymbol{T},_{\alpha}
\end{gathered}
$$

where the tensor $\boldsymbol{T}$ is given as the decomposition defined in $\mathrm{Eq}(13)$. Since $\boldsymbol{g}_{3}$ is assumed normal to the vectors $\boldsymbol{g}_{\alpha}$ and $\boldsymbol{g}_{\beta}$, we have that:

$$
\boldsymbol{g}_{3}=\frac{\boldsymbol{g}_{\alpha} \times \boldsymbol{g}_{\beta}}{\left\|\boldsymbol{g}_{\alpha} \times \boldsymbol{g}_{\beta}\right\|}
$$

and

$$
\begin{gathered}
\boldsymbol{g}_{\alpha} \cdot \boldsymbol{g}_{3}=g_{\alpha 3}=0 \\
\boldsymbol{g}_{3} \cdot \boldsymbol{g}_{3}=1 \\
\boldsymbol{g}_{3, \alpha} \cdot \boldsymbol{g}_{3}=0 \\
\boldsymbol{g}_{3,3} \cdot \boldsymbol{g}_{3}=0
\end{gathered}
$$


Using Eqs(20-21) the metric tensor components in matrix form are:

$$
g_{i j}=\left[\begin{array}{ccc}
g_{11} & g_{12} & 0 \\
g_{21} & g_{22} & 0 \\
0 & 0 & 1
\end{array}\right]
$$

It is well known that when a base vector is differentiated with respect to general curvilinear coordinates, the resulting expressions can be written as components of either a covariant or a contravariant base vector. Here the Christoffel symbols of the first and second kind are introduced respectively and defined as:

$$
\begin{gathered}
\Gamma_{i j k}=\boldsymbol{g}_{i, j} \cdot \boldsymbol{g}_{k} \\
\Gamma_{i j}^{k}=\boldsymbol{g}_{i, j} \cdot \boldsymbol{g}^{k}
\end{gathered}
$$

It follows from Eqs (22-23) that :

$$
\Gamma_{3 \alpha 3}=\Gamma_{\alpha 33}=\Gamma_{33 \alpha}=\Gamma_{333}=0
$$

Differentiating $\operatorname{Eq}(20)$ with respect to $\beta$ index yields:

$$
\boldsymbol{g}_{\alpha, \beta} \cdot \boldsymbol{g}_{3}+\boldsymbol{g}_{3, \beta} \cdot \boldsymbol{g}_{\alpha}=0
$$

This together with $\mathrm{Eq}(24)$ gives the following relations for the Christoffel symbols of the first kind:

$$
\Gamma_{\alpha \beta 3}=-\Gamma_{3 \beta \alpha}=-\Gamma_{\beta 3 \alpha}=\Gamma_{\beta \alpha 3}=-\Gamma_{3 \alpha \beta}=-\Gamma_{\alpha 3 \beta}
$$

It is known that the third index of the Christoffel symbol can be lowered and raised by multiplying by the metric tensor. Using this fact together with Eq (26) yields:

$$
\Gamma_{3 \alpha}^{3}=\Gamma_{33}^{\alpha}=\Gamma_{33}^{3}=0
$$


The covariant derivatives of arbitrary vectors $u^{i}$ and $u_{i}$ are given by $u^{i}{ }_{j}+u^{k} \Gamma_{k j}^{i}$ and $u_{i, j}-u_{k} \Gamma_{i j}^{k}$ respectively.

For the representation given by $\mathrm{Eq}(17)$ the following relationships follow for the covariant derivatives of a vector component $u_{\alpha}$ where Eqs(28-29) have been used:

$$
\begin{gathered}
u_{\alpha^{\prime} \beta}=u_{\alpha, \beta}-u_{\gamma} \Gamma_{\alpha \beta}^{\gamma}-u_{3} \Gamma_{\alpha \beta}^{3} \\
u_{\alpha^{\prime} 3}=u_{\alpha, 3}-u_{\gamma} \Gamma_{\alpha 3}^{\gamma} \\
u_{3^{\prime} \alpha}=u_{3, \alpha}-u_{\gamma} \Gamma_{3 \alpha}^{\gamma} \\
u_{\left.\right|_{\beta}}^{\alpha}=u^{\alpha}, \beta+u^{\gamma} \Gamma_{\beta \gamma}^{\alpha}+u^{3} \Gamma_{\beta 3}^{\alpha} \\
u_{\left.\right|_{3}}^{\alpha}=u^{\alpha}{ }_{3}+u^{\gamma} \Gamma_{\gamma 3}^{\alpha} \\
u_{\left.\right|_{\alpha}}^{3}=u^{3},_{\alpha}+u^{\gamma} \Gamma_{\alpha \gamma}^{3}
\end{gathered}
$$

Now the base vectors when differentiated with respect to the coordinates indexed by $i$ the following are known to be true:

$$
\boldsymbol{g}_{i, j}=\Gamma_{i j}^{k} \boldsymbol{g}_{k}=\Gamma_{i j k} \boldsymbol{g}^{k}
$$

On the surface of the manifold we may write:

$$
\boldsymbol{T}_{3, \alpha}=\Gamma_{3 \alpha \beta} \boldsymbol{T}^{\beta}+\Gamma_{3 \alpha 3} \boldsymbol{T}^{3}
$$

Using $\operatorname{Eqs}(26)$ and (28) the above expression can be simplified to:

$$
\boldsymbol{T}_{3, \alpha}=-\Gamma_{\alpha \beta 3} \boldsymbol{T}^{\beta}
$$


As pointed out in [11] the term $\Gamma_{\alpha \beta 3}$ is a measure of the rate of change of the base vector $\boldsymbol{T}_{3}$ along a parametric curve. $b_{\alpha \beta}=\Gamma_{\alpha \beta 3}$ is a plane symmetric tensor and is referred to as the curvature tensor. We have:

$$
b_{\alpha \beta}=-\boldsymbol{T}_{3, \alpha} \cdot \boldsymbol{T}_{\beta}
$$

From Eq (27), on the surface of the manifold, the curvature tensor may be written as:

$$
b_{\alpha \beta}=\boldsymbol{T}_{\alpha, \beta} \cdot \boldsymbol{T}_{3}=-\boldsymbol{T}_{3, \beta} \cdot \boldsymbol{T}_{\alpha}
$$

The mixed and contravariant components of the curvature tensor can be obtained by using the metric tensor. Let:

$$
\boldsymbol{u}=u_{\alpha} \boldsymbol{T}^{\alpha}+u_{3} \boldsymbol{T}^{3}
$$

Differentiating $\boldsymbol{u}$ with respect to $\beta$ and using covariant derivative and Eqs(30-32) yields:

$$
\boldsymbol{u}{,{ }_{\beta}}=u_{\alpha^{\prime}{ }_{\beta}} \boldsymbol{T}^{\alpha}+u_{3_{\beta}{ }^{\prime}} \boldsymbol{T}^{3}=\left(u_{\alpha, \beta}-u_{\gamma} \Gamma_{\alpha \beta}^{\gamma}-u_{3} b_{\alpha \beta}\right) \boldsymbol{T}^{\alpha}+\left(u_{3, \beta}+u_{\gamma} b_{\beta}^{\gamma}\right) \boldsymbol{T}^{3}
$$

Let

$$
u_{\alpha \|_{\beta}}=u_{\alpha, \beta}-u_{\gamma} \Gamma_{\alpha \beta}^{\gamma}
$$

Eq (42) is therefore simplified yielding:

$$
\boldsymbol{u},_{\beta}=\left(u_{\alpha^{\prime \prime} \beta}-u_{3} b_{\alpha \beta}\right) \boldsymbol{T}^{\alpha}+\left(u_{3, \beta}+u_{\gamma} b_{\beta}^{\gamma}\right) \boldsymbol{T}^{3}
$$

The covariant derivative of $u_{\alpha}$ can therefore be written in the form

$$
u_{\alpha \beta}=u_{\alpha^{\|} \beta}-u_{3} b_{\alpha \beta}
$$

In a similar fashion, if the vector $\boldsymbol{u}$ is defined in terms of its contravariant components the following relationships hold: 


$$
\begin{gathered}
u_{\|_{\beta}}^{\alpha}=u_{, \beta}^{\alpha}+u^{\gamma} \Gamma_{\beta \gamma}^{\alpha} \\
u_{\left.\right|_{\beta}}^{\alpha}=u_{\|_{\beta}}^{\alpha}-u^{3} b_{\beta}^{\alpha} \\
\boldsymbol{u},_{\beta}=\left(u_{\|_{\beta}}^{\alpha}-u^{3} b_{\beta}^{\alpha}\right) \boldsymbol{T}_{\alpha}+\left(u^{3},_{\beta}+u^{\gamma} b_{\gamma \beta}\right) \boldsymbol{T}_{3}
\end{gathered}
$$

For points adjacent to the surface of the manifold $\boldsymbol{u}$ is also defined. The partial derivative of $\boldsymbol{u}$ with respect to the variable in the normal direction $\boldsymbol{N}$ exists and can be expressed as a function of the contravariant or covariant base vectors as follows:

$$
\begin{gathered}
\boldsymbol{u}_{3}=u_{\alpha^{\prime}{ }_{3}} \boldsymbol{T}^{\alpha}+u_{3{ }_{3}} \boldsymbol{T}^{3}=\left(u_{\alpha, 3}+u_{\gamma} b_{\alpha}^{\gamma}\right) \boldsymbol{T}^{\alpha}+u_{3,3} \boldsymbol{T}^{3} \\
\boldsymbol{u}_{3}=u_{\left.\right|_{3}}^{\alpha} \boldsymbol{T}_{\alpha}+u_{\left.\right|_{3}}^{3} \boldsymbol{T}_{3}=\left(u^{\alpha}{ }_{3}-u^{\gamma} b_{\gamma}^{\alpha}\right) \boldsymbol{T}_{\alpha}+u^{3}{ }_{3} \boldsymbol{T}_{3}
\end{gathered}
$$

\section{CMS Surface Analysis}

For the parametrization with arguments omitted, it was already established a dynamic surface with vibrations occuring can be parametrized as:

$$
\mathbf{R}^{*}=\mathbf{R}+\sigma^{\mu} \boldsymbol{\xi}_{\mu}
$$

\subsection{Vibrating Surface Shift Tensor}

For the surface, we first analyze the new Tangent Vectors, $\mathbf{S}_{\alpha}^{*}$, which is an indicial method of notating the Tangent Space of a manifold:

$$
\begin{aligned}
& \mathbf{S}_{\alpha}^{*}=\left(\mathbf{R}^{*}\right)_{, \alpha} \\
& \mathbf{S}_{\alpha}^{*}=\left(\mathbf{R}+\sigma^{\mu} \boldsymbol{\xi}_{\mu}\right)_{, \alpha} \\
& \mathbf{S}_{\alpha}^{*}=\mathbf{S}_{\alpha}+\left(\sigma_{, \alpha}^{\mu}+\sigma^{\nu} \tilde{\Gamma}_{\alpha \nu}^{\mu}\right) \boldsymbol{\xi}_{\mu}
\end{aligned}
$$


From section 2, the expression in paranthesies can be abbreviated in a sort of covariant derivative:

$$
\mathbf{S}_{\alpha}^{*}=\mathbf{S}_{\alpha}+\left(\tilde{\nabla}_{\alpha} \sigma^{\mu}\right) \boldsymbol{\xi}_{\mu}
$$

Therefore, since we know that the Surface Frame Basis is expressable in terms of the ambient basis $\boldsymbol{\xi}_{\mu}=\zeta_{\mu}^{i} \mathbf{Z}_{i}$ and since the Surface Basis in the deformed and non deformed case is expressable as the following: $\mathbf{S}_{\alpha}=Z_{\alpha}^{i} \mathbf{Z}_{i}$, then the equation is also expressable in its complete tensor notation as:

$$
Z_{\alpha}^{i *}=Z_{\alpha}^{i}+\left(\tilde{\nabla}_{\alpha} \sigma^{\mu}\right) \zeta_{\mu}^{i}
$$

This indicial method of notating the tangent space with respect to the ambient basis is noted as the shift tensor and here is the post-vibration shift tensor of the manifold. This is indeed a tensor as can be easily confirmed by analyzing that each of the terms of the expression is an individual tensor with respect to its basis space.

\subsection{Vibrating Surface Metric Tensor}

We now turn to the surface metric tensor, $S_{\alpha \beta}^{*}$. Intuitively, we define that for such a shift tensor, the metric must be given as [1]:

$$
S_{\alpha \beta}^{*}=Z_{i j} Z_{\alpha}^{i *} Z_{\beta}^{j *}
$$

After expanding the definition of the new tangent vector components, and using the distributive property of multiplication, the new metric tensor is obtained to be defined 
in terms of the displacement's components, $\sigma^{\mu}$ and original metric tensor as:

$$
S_{\alpha \beta}^{*}=S_{\alpha \beta}+Z_{i j} Z_{\alpha}^{i} \zeta_{\nu}^{j} \tilde{\nabla}_{\beta} \sigma^{\nu}+Z_{i j} Z_{\beta}^{j} \zeta_{\mu}^{i} \tilde{\nabla}_{\alpha} \sigma^{\mu}+Z_{i j} \zeta_{\mu}^{i} \zeta_{\nu}^{j} \tilde{\nabla}_{\alpha} \sigma^{\mu} \tilde{\nabla}_{\beta} \sigma^{\nu}
$$

The tensor can be simplified by expanding the displacement operators and contracting indices where appropriate. The equation after simplification has a final form given by:

$$
S_{\alpha \beta}^{*}=S_{\alpha \beta}+2 \tilde{\nabla}_{\left({ }_{\alpha} \sigma_{\beta}\right)}+\xi_{\mu \nu} \tilde{\nabla}_{\alpha} \sigma^{\mu} \tilde{\nabla}_{\beta} \sigma^{\nu}
$$

This can also be expressed as:

$$
S_{\alpha \beta}^{*}=S_{\alpha \beta}+2 \tilde{\nabla}_{\left(\alpha \sigma_{\beta}\right)}+\tilde{\nabla}_{\alpha} \overrightarrow{\boldsymbol{\sigma}} \cdot \tilde{\nabla}_{\beta} \overrightarrow{\boldsymbol{\sigma}}
$$

This is nonlinear operator with respect to the displacement's components, $\sigma^{\mu}$. It is also a special instance of an operator, which contains the first, second, and third groundforms all in one. It is worth to note that if there are no vibrations, ie $\sigma^{\mu}=0$, then we obtain the expected equivalency:

$$
S_{\alpha \beta}^{*}=S_{\alpha \beta}
$$

\subsection{Vibrating Surface Normal}

By analogue of the original definition of the normal in terms of the shift tensors, we state that the post-vibration normal will be given by:

$$
\mathbf{N}^{*}=\frac{1}{2} \mathbf{Z}^{i} \epsilon_{i j k} \epsilon^{\alpha \beta} Z_{\alpha}^{j *} Z_{\beta}^{k *}
$$

We expand out the equation in terms of the expansion of the shift tensors, noticing

that by symmetry, the middle two terms can be abbreviated into the same term and 
find that:

$$
\mathbf{N}^{*}=\mathbf{N}+\mathbf{Z}^{i} \epsilon_{i j k} \epsilon^{\alpha \beta} Z_{\alpha}^{j} \zeta_{\nu}^{k} \tilde{\nabla}_{\beta} \sigma^{\nu}+\mathbf{Z}^{i} \frac{1}{2} \epsilon_{i j k} \epsilon^{\alpha \beta} \zeta_{\mu}^{j} \zeta_{\nu}^{k} \tilde{\nabla}_{\alpha} \sigma^{\mu} \tilde{\nabla}_{\beta} \sigma^{\nu}
$$

This equation can be grouped into the following:

$$
\mathbf{N}^{*}=\mathbf{N}+\mathbf{Z}^{i} \epsilon_{i j k} \epsilon^{\alpha \beta} \zeta_{\nu}^{k} \tilde{\nabla}_{\beta} \sigma^{\nu}\left(Z^{j}{ }_{\alpha}+\frac{1}{2} \zeta^{j}{ }_{\mu} \tilde{\nabla}_{\alpha} \sigma^{\mu}\right)
$$

\section{Invariance of the Normal}

As expected, we see that if there are no deformations by waves, ie. if $\sigma^{\mu}=0$, then we will obtain the expected equivalency:

$$
\mathbf{N}^{*}=\mathbf{N}
$$

There is another additional case worth mentioning. We see that if there exists a surface which abides by the equation:

$$
2 Z_{\alpha}^{j}+\zeta_{\mu}^{j} \tilde{\nabla}_{\alpha} \sigma^{\mu}=0
$$

Then its normal will stay unchanged under a motion of the surface. This equation can be simplified remembering that $Z_{\alpha}^{i *}=Z_{\alpha}^{i}+\left(\tilde{\nabla}_{\alpha} \sigma^{\mu}\right) \zeta_{\mu}^{i}$ and grouping the term out of the equation:

$$
Z_{\alpha}^{j *}+Z_{\alpha}^{j}=0
$$

Or it can also be contracted with the ambient basis to give the following form:

$$
\mathbf{S}_{\alpha}+\mathbf{S}_{\alpha}^{*}=0
$$

This equation much like many equations in CMS is fully analyzed by observing its normal and tangential components. If we consider the equation's tangential components, 
then we see that the following equality can be obtained:

$$
\begin{aligned}
2 Z^{j}{ }_{\alpha} Z^{\beta}{ }_{j}+Z^{\beta}{ }_{j}{ }^{j}{ }_{\mu} \tilde{\nabla}_{\alpha} \sigma^{\mu} & =0 \\
2 \delta_{\alpha}^{\beta}+Z^{\beta}{ }_{j} Z^{j}{ }_{\gamma} \tilde{\nabla}_{\alpha} \sigma^{\gamma} & =0 \\
2 \delta_{\alpha}^{\beta}+\delta_{\gamma}^{\beta} \tilde{\nabla}_{\alpha} \sigma^{\gamma} & =0 \\
2 \delta_{\alpha}^{\beta}+\tilde{\nabla}_{\alpha} \sigma^{\beta} & =0
\end{aligned}
$$

Separating the displacement into its tangential and normal components explicitly $\sigma^{\mu} \boldsymbol{\xi}_{\mu}=\sigma^{\alpha} \mathbf{S}_{\alpha}+\sigma^{3} \mathbf{N}$, then we see that the equation reduces:

$$
\begin{array}{r}
2 \delta_{\alpha}^{\beta}+\sigma_{, \alpha}^{\beta}+\tilde{\Gamma}_{\alpha \mu}^{\beta} \sigma^{\mu}=0 \\
2 \delta_{\alpha}^{\beta}+\sigma_{, \alpha}^{\beta}+\tilde{\Gamma}_{\alpha \gamma}^{\beta} \sigma^{\gamma}+\tilde{\Gamma}_{\alpha 3}^{\beta} \sigma^{3}=0 \\
2 \delta_{\alpha}^{\beta}+\sigma_{, \alpha}^{\beta}+\tilde{\Gamma}_{\alpha \gamma}^{\beta} \sigma^{\gamma}-B_{\alpha}^{\beta} \sigma^{3}=0
\end{array}
$$

And so the tangential component of the equation reduces to the following final form:

$$
2 \delta_{\alpha}^{\beta}+\nabla_{\alpha} \sigma^{\beta}-B_{\alpha}^{\beta} \sigma^{3}=0
$$

In a similar fashion, the normal component of the equation reduces to the following final form:

$$
\nabla_{\alpha} \sigma^{3}+B_{\alpha \beta} \sigma^{\beta}=0
$$

These therefore will compose a complicated system of equations for the components. If the normal of a surface is to remain stable under a displacement motion, the displacement must be in the following form and satisfy the following equations: 


$$
\left\{\begin{array}{c}
2 \delta_{\alpha}^{\beta}+\nabla_{\alpha} \sigma^{\beta}-B_{\alpha}^{\beta} \sigma^{3}=0 \\
\nabla_{\alpha} \sigma^{3}+B_{\alpha \beta} \sigma^{\beta}=0
\end{array}\right.
$$

These equations are completely tensorial and in fact have much geometric meaning endowed within them. This can be seen by considering a few simple cases for equations.

\section{A Purely Normal Displacement}

If we assume that $\sigma^{\alpha}=0$ and thus the displacement will have the following form, $\sigma^{\mu} \boldsymbol{\xi}_{\mu}=\sigma^{3} \mathbf{N}$, then we see that the set of equations reduces to the following much more simple set:

$$
\left\{\begin{array}{c}
2 \delta_{\alpha}^{\beta}-B_{\alpha}^{\beta} \sigma^{3}=0 \\
\nabla_{\alpha} \sigma^{3}=0
\end{array}\right.
$$

This set can be easily solved by first considering the first equation. Since here, the way that $\sigma^{3}$ transforms is in an invariant fashion, then we can see that it implies that $\sigma^{3}=$ constant. In the spirit of CMS, we will take a constant to be a constant with respect to the surface variables, meaning it can still be dependent on time, $\sigma^{3}(t)$. In this case, the first equation reduces to the following identity:

$$
2 \delta_{\alpha}^{\beta}=B_{\alpha}^{\beta} \sigma^{3}
$$

Isolating this for the curvature tensor, we see that it must adhere to following form:

$$
B_{\alpha}^{\beta}=\frac{2}{\sigma^{3}(t)} \delta_{\alpha}^{\beta}
$$


For the right form of $\sigma^{3}$, this is the dependent form of the curvature tensor for a sphere.

$$
B_{\alpha}^{\beta}= \pm\left[\begin{array}{cc}
\frac{1}{R} & 0 \\
0 & \frac{1}{R}
\end{array}\right]
$$

where $R$ is its radius. Therefore what our analysis for a purely normal displacement implies is that a Normal will remain rigid if its curvature tensor is in the form of a diagonal matrix with identical entries that do not depend on the surface, but may vary in time.

In this specific application, it relates the intuitive fact that a sphere which is expanding at any any rate uniformly in the direction of its normal will preserve its normal; this is as expected. Now, however if we consider a slightly weaker case of the solution where we ignore the second equation because the displacement field varies very slightly spatially such that the second equation is satisfied to a small order then we see that only the first equation remains which can be contracted into the following form:

$$
\sigma^{3}=\frac{4}{B_{\alpha}^{\alpha}}
$$

We will name this the curvature displacement equation. Thus, we see that a surface will approximately allow its normal to stay rigid and stable if its mean curvature is proportional to the inverse of its displacement due to vibrations. Again, in keeping with the spirit of CMS, a fasciinating property of the equation is that it also implies a direct time dependence on both terms:

- If the displacement is stationary, the curvature required to counteract any change in normals, too, must be referring to a surface who is stationary.

- Vice-versa, if the surface has a curvature which is time-independent, any displacements, too, will be stationary.

- If the displacement is dynamic, the curvature of the surface required to counteract 
any vibrations must also be time-dependent and therefore be referring to a surface which is moving.

- In addition, a dynamic surface automatically implies that any displacement required to counteract such motion will be time dependent too.

\subsection{Impossibility of a Purely Tangential Motion}

Here we will examine the motivation for why a purely tangential motion is impossible to maintain a normal of a surface. In the case we are considering a purely tangential motion, $\sigma^{\mu} \boldsymbol{\xi}_{\mu}=\sigma^{\alpha} \mathbf{S}_{\alpha}$, where we would consider $\sigma^{3}=0$, then we see that the equations reduce to the following form:

$$
\left\{\begin{array}{c}
2 \delta_{\alpha}^{\beta}+\nabla_{\alpha} \sigma^{\beta}=0 \\
B_{\alpha \beta} \sigma^{\beta}=0
\end{array}\right.
$$

If we consider the second equation, since an inverse can always be found, it automatically implies that $\sigma^{\beta}=0$. Hoping that this trivial solution will satisfy the first equation , we see that the covariant derivative of a zero vector reduces to the following equality:

$$
\delta_{\alpha}^{\beta}=0
$$

Since the Kronecker Delta implies that the diagonal entries must be 1, this implies an inequality for two out of the 4 entries. Thus this establishes that it is impossible to find a tangential motion which will preserve the normal of a surface.

\subsection{Addressing the Completely General Case}

The completely general case is significantly more difficult to work with, but still able to work with. We review the general case of equations: 


$$
\left\{\begin{array}{c}
2 \delta_{\alpha}^{\beta}+\nabla_{\alpha} \sigma^{\beta}-B_{\alpha}^{\beta} \sigma^{3}=0 \\
\nabla_{\alpha} \sigma^{3}+B_{\alpha \beta} \sigma^{\beta}=0
\end{array}\right.
$$

The first step is to postulate the existence of an inverse of the curvature tensor which is given by:

$$
\hat{B}^{\alpha \beta}=\frac{1}{K} \epsilon^{\alpha \beta} \epsilon^{\gamma \delta} B_{\beta \delta}
$$

where $K$ is the determinant of the curvature tensor, $B_{\beta}^{\alpha}$ and is known as the Gaussian curvature of the surface. Most importantly, the Inverse curvature tensor has the following property:

$$
\hat{B}^{\alpha \beta} B_{\beta \gamma}=\delta_{\gamma}^{\alpha}
$$

Using this property, we can isolate the second equation for $\sigma^{\alpha}$ :

$$
\sigma^{\gamma}=-\hat{B}^{\gamma \alpha} \nabla_{\alpha} \sigma^{3}
$$

This can be substituted into the first equation to obtain $\sigma^{3}$. This resultant PDE is a second order symmetric, linear non-homogeneous, non-autonomous tensor equation:

$$
\left(\nabla_{\alpha} \nabla_{\beta}--\hat{B}^{\gamma \delta}\left(\nabla_{\gamma} B_{\alpha \beta}\right) \nabla_{\delta}+\tau_{\alpha \beta}\right) \sigma^{3}=2 B_{\alpha \beta}
$$

The symmetry of the equation is generated by the symmetry of the curvature tensors, the square curvature tensor and the identity that for an Invariant, covariant derivatives commute. Once this solution is obtained, it will be in terms of the curvature tensor. We see that it can be substituted back into the second equation to obtain $\sigma^{\alpha}$ and once these are obtained, $\overrightarrow{\boldsymbol{\sigma}}$ can be reconstructed and the only unknown for dynamic vibrations is to satisfy the wave equation: 


$$
\tilde{\square}|\overrightarrow{\boldsymbol{\sigma}}|=0
$$

Thus this will allow us to obtain the curvature tensor, $B_{\alpha \beta}$, and thus with the solved equations, when we have $|\overrightarrow{\boldsymbol{\sigma}}|$ and $B_{\alpha \beta}$, these will specify the equation from which the deformation is occurring.

\subsection{Extending a Purely Normal Motion Analysis}

We reflect on the Curvature-Proportionality Relation, which states that to have an invariance of the normal at each point on a surface during dynamics vibrations, curvature and the normal displacement must be related as such:

$$
\sigma^{3} B_{\alpha}^{\alpha}=4
$$

We can see that in the special case that the displacement is purely normal, then $|\overrightarrow{\boldsymbol{\sigma}}|=\sigma^{3}$. This solution is unique as it is also coupled to the form of dynamic waves on the surface by the surface wave equation as:

$$
\tilde{\square} \sigma^{3}=\left(\frac{1}{c^{2}} \frac{\partial^{2}}{\partial t^{2}}-\nabla_{\beta} \nabla^{\beta}\right) \sigma^{3}=0
$$

We can relate these two equations by stating that in the surface wave equation, we will have $\sigma^{3}=f\left(B_{\alpha}^{\alpha}\right)$. In our case, we have $f(x)=4 x^{-1}$. This will nonlinearize the wave equation and the solution will be the curvature of the mystery surface.

The equation is interesting since the equation will depend on the Laplacian of the mean curvature of the surface which entirely defines the surface Laplacian. Thus the two equations together can be represented as:

$$
\tilde{\square} f\left(B_{\alpha}^{\alpha}\right)=0
$$


After expanding the form using the exact definition of the surface Laplacian and allowing the chain rules to be implemented, the form of the equation is reduced to:

$$
\frac{1}{c^{2}} \frac{\partial}{\partial t}\left(\frac{d f}{d B_{\alpha}^{\alpha}} \frac{\partial B_{\alpha}^{\alpha}}{\partial t}\right)-\frac{1}{\sqrt{\mid S_{\omega \lambda \mid}}} \frac{\partial}{\partial S^{\beta}}\left(\sqrt{\left|S_{\omega \lambda}\right|} S^{\beta \gamma} \frac{d f}{d B_{\alpha}^{\alpha}} \frac{\partial B_{\alpha}^{\alpha}}{\partial S^{\gamma}}\right)=0
$$

Expanding with the chain rule, factoring, and reintroducing differential objects, we obtain:

$$
\frac{d f}{d B_{\alpha}^{\alpha}}\left[\frac{1}{c^{2}} \frac{\partial^{2} B_{\alpha}^{\alpha}}{\partial t^{2}}-\nabla_{\beta} \nabla^{\beta} B_{\alpha}^{\alpha}\right]+\frac{d^{2} f}{d\left(B_{\alpha}^{\alpha}\right)^{2}}\left[\frac{1}{c^{2}}\left(\frac{\partial B_{\gamma}^{\gamma}}{\partial t}\right)^{2}-S^{\beta \gamma}\left(\nabla_{\beta} B_{\alpha}^{\alpha}\right)\left(\nabla_{\gamma} B_{\alpha}^{\alpha}\right)\right]=0
$$

Using $\left({ }^{*}\right)$ to denote time derivatives, recognizing the surface D'Alembert operator, raising indices on the surface covariant derivatives, and noticing that $\left(\nabla^{\beta} B_{\alpha}^{\alpha}\right)\left(\nabla_{\beta} B_{\alpha}^{\alpha}\right)$ can be expressed as the squared magnitude of the mean curvature's gradient. We continue simplification, and finally obtain:

$$
\frac{1}{c^{2}}\left(\dot{B_{\alpha}^{\alpha}}\right)^{2}-\left|\nabla B_{\alpha}^{\alpha}\right|^{2}=-\left[\frac{d^{2} f}{d\left(B_{\alpha}^{\alpha}\right)^{2}}\right]^{-1} \frac{d f}{d B_{\alpha}^{\alpha}} \tilde{\square} B_{\alpha}^{\alpha}
$$

Where $\nabla=\mathbf{S}^{\beta} \nabla_{\beta}=\mathbf{S}_{\beta} \nabla^{\beta}$. Introducing our reciprocal function, we finally simplify the equation as:

$$
\frac{1}{c^{2}}\left(\dot{B}_{\alpha}^{\alpha}\right)^{2}-\left|\nabla B_{\alpha}^{\alpha}\right|^{2}=\frac{1}{2} B_{\alpha}^{\alpha} \tilde{\square} B_{\alpha}^{\alpha}
$$

We denote this equation as the Invariant-Normal Curvature Wave Equation. Notice how it is non-linear in all terms. In addition, the equation appears to be completely autonomous.

\subsection{Trivial Solutions to the Invariant-Normal Curvature Wave Equation}

This equation admits several interesting trivial solutions 


\section{Minimal Surface}

Immediately, we notice that $B_{\alpha}^{\alpha}=0$ is a mathematical solution to the equation, since the equation is autonomous in nature. Vanishing mean curvature usually characterizes minimal surfaces.

The only issue with this definition is that substituting the solution into the curvature-perturbation equality:

$$
\sigma^{3}=\frac{4}{B_{\alpha}^{\alpha}}
$$

results in waves with an undefined wave deformation magnitude; an inadmissible solution.

\section{Constant Curvature}

In addition, considering constant solutions, we notice how a constant curvature will also produce constant wave perturbations. These will be constant according to

$$
\sigma^{3}=\frac{4}{B_{\alpha}^{\alpha}}
$$

This solution is trivial since this refers to stationary wave perturbations which enlarge the local radius of the surface.

- An object with constant mean curvature is the sphere.

- The equality states that in order to have an invariance of the normal, waves will enlarge the surface.

- This is the equivalent of enlarging the radius of the sphere which, as expected, will not change the normal. 


\subsection{Invariant-Normal Curvature Wave Equation on a Surface of Revolution}

This section pertains to applying the equation to the curvature of a surface of revolution. Here, the surface is parametrized by:

$$
\left\{\begin{array}{c}
x=R \cos \theta \\
y=R \sin \theta \text { where, } \mathrm{R}=\mathrm{R}(\mathrm{z}, \mathrm{t}) \\
z=z
\end{array}\right.
$$

In here, the solution to the equation will be the unknown $R$ which will be the z- \& time-dependent radius of the surface or revolution.

This surface of revolution, $R$, will produce an invariant normal after the application of any wave perturbations determined by its surface Laplacian. After it is established that $\frac{\partial}{\partial z} R=R_{z}, \frac{\partial^{2}}{\partial z^{2}} R=R_{z z}$, and $\lambda=\sqrt{R_{z}^{2}+1}$, calculating all the descriptors, metrics, groundforms, the mean curvature of the surface of revolution is found to be:

$$
B_{\alpha}^{\alpha}=\frac{R R_{z z}-\lambda^{2}}{R \lambda^{3}}=\frac{R R_{z z}-R_{z}^{2}-1}{R\left(R_{z}^{2}+1\right)^{\frac{3}{2}}}
$$

\section{Differential Operator Objects}

Based on these objects we find that the covariant and contravariant derivatives of a scalar function, $\phi$, is:

$$
\nabla_{\alpha} \phi=\left[\begin{array}{ll}
\frac{\partial \phi}{\partial \theta} & \frac{\partial \phi}{\partial z}
\end{array}\right], \nabla^{\alpha} \phi=\left[\begin{array}{cc}
\frac{1}{R^{2}} \frac{\partial \phi}{\partial \theta} & \frac{1}{\lambda^{2}} \frac{\partial \phi}{\partial z}
\end{array}\right]
$$

Therefore, its magnitude of the gradient of $\phi$, if $\phi=\phi(z, t)$ (as it is for the curvature) is given by:

$$
|\nabla \phi|^{2}=\frac{1}{\lambda^{2}}\left(\frac{\partial \phi}{\partial z}\right)^{2}
$$


The same $\phi=\phi(z, t)$ also admits a surface Laplacian as:

$$
\nabla_{\alpha} \nabla^{\alpha} \phi=\frac{1}{\lambda R} \frac{\partial}{\partial z}\left(\frac{R}{\lambda} \frac{\partial \phi}{\partial z}\right)
$$

\section{The Invariant-Normal Curvature Wave Equation}

Incorporating all the elements into the equation, we obtain the surface of revolution invariant-normal curvature wave equation:

$$
\frac{1}{c^{2}}\left(\frac{\partial B_{\alpha}^{\alpha}}{\partial t}\right)^{2}-\frac{1}{\lambda^{2}}\left(\frac{\partial B_{\alpha}^{\alpha}}{\partial z}\right)^{2}=\frac{1}{2 c^{2}} B_{\alpha}^{\alpha} \frac{\partial^{2} B_{\alpha}^{\alpha}}{\partial t^{2}}-\frac{1}{2 \lambda R} B_{\alpha}^{\alpha} \frac{\partial}{\partial z}\left(\frac{R}{\lambda} \frac{\partial B_{\alpha}^{\alpha}}{\partial z}\right)
$$

where,

$$
B_{\alpha}^{\alpha}=\frac{R R_{z z}-\lambda^{2}}{R \lambda^{3}}, \lambda^{2}=R_{z}^{2}+1, \text { and } R=R(z, t)
$$

An immediate, time-independent solution is the case where $R(z, t)=c$ (corresponding to a cylinder). This is significant since it has a constant curvature, and therefore reduces the equation to null.

- By the curvature-wave perturbation equality, this will result in stationary perturbations enlarging the radius of the cylinder (a trivial solution)

The stationary catenoid also satisfies the equation, having globally vanishing mean curvature (as seen by satisfying the equation)

$$
R R_{z z}-\lambda^{2}=0
$$

However, having zero mean curvature, it will also produce stationary waves of an inadmissible form. The equation can also be separated if special cases are considered for the morphology of the surface. 


\section{Conclusion}

Therefore a framework for analyzing dynamic vibrations is established on arbitrary surfaces embedded in an arbitrary ambient space. Using the framework, a decomposition of the post-vibrational surface is made in terms of the normal of a surface. Restricting the study to the Cartesian ambient space, an expression for the shift tensor, metric tensor, and normal of such a surface were explicitly obtained. Conditions were obtained on the form of the dynamic vibrations under which the normal of the surface will equalize any initial motions using the dynamic vibrations producing the curvatureperturbation equality. The equality is applied to the surface wave equation to obtain the Invariant Normal Curvature Wave Equation. An equation has been explicitly formulated in terms of a surface of revolution and trivial solution were encountered

The fundamental invariant normal curvature wave equation represents a differential form of an algorithm which can potentially be applied to stabilization applications outlined earlier in cellular biology and seismic science. The theoretical framework that the analysis of dynamic vibrations yields has much application beyond stabilization algorithms. It can be used in the future to relate metabolics of cells and actin dynamics to the imposed vibrations which are superimposed on the already dynamic cellular phospholipid bilayer surface 


\section{References}

[1] P.Grinfeld, Introduction to tensor analysis and the calculus of moving surfaces, Springer 2010.

[2] R.G. Jacquot,W. Soedel, Vibrations of Elastic Surface Systems Carrying Dynamic Elements, The Journal of the Acoustical Society of America, Vol 47 No.5(Part 2), 1970.

[3] Philip D. Cha, Free vibration of a uniform beam with multiple elastically mounted two-degree-of-freedom systems, doi:10.1016/j.jsv.2007.06.063, 2007, pp 1-7.

[4] E.H. Dowell, The Behaviour of a Linear, Damped Modal System with a NonLinear Spring-Mass-Dry Friction Damper System Attached, Journal of Sound and Vibration, 89(1), 1983, pp 65-84.

[5] W. Soedel, Vibrations of Shells and Plates, Marcel Dekker Inc. , 3rd Edition 2004

[6] J. J. de Espindola, Carlos Alberto Bavastri and Eduardo Marcio de Oliveira Lopes, On the Design of Optimum System of Viscoelastic Vibration Absorbers for a Given Viscoelastic Material,Proceedings of the XII International Symposium on Dynamic Problems of Mechanics (DINAME 2007), Varoto, P. S. and Trindade, M. A. (Editors), ABCM, Ilhabela, SP, Brazil, February 26 - March 2, 2007

[7] J. Bereiter-Hahn, O.R. Anderson, W.-E.Reif (Eds.), Cytomechanics: The Mechanical Basis of Cell Form and Structure, Springer-Verlag, 1987.

[8] I.S.Sokolnikoff, Tensor Analysis, Theory and applications,New York-John Wiley and Sons, Inc. London-Chapman and Hall, Limited, 1951.

[9] R.G. Jacquot, The Spatial Average Mean Square Motion As an Objective Function for Optimizing Damping in Damped Modified Systems, Journal of Sound and Vibration 259(4), 2003, pp 955-965. 
[10] R. G. Jacquot, Suppression of random vibration in plates using vibration absorbers, Journal of Sound and Vibration, 248, pp 585 - 596, 2001.

[11] S. Naomis, P.C.M. Lau, Computational Tensor Analysis of Shell Structures, Lecture Notes in Engineering 58, Edited by C.A. Brebbia and S.A. Orszag, 1990. 